\title{
Some Inconsistencies in NICE's Consideration of Social Values
}

\author{
Mike Paulden • James F. O'Mahony • \\ Anthony J. Culyer $\cdot$ Christopher McCabe
}

Published online: 22 August 2014

(C) Springer International Publishing Switzerland 2014

\begin{abstract}
The UK's National Institute for Health and Care Excellence (NICE) recently proposed amendments to its methods for the appraisal of health technologies. Previous amendments in 2009 and 2011 placed a greater value on the health of patients at the "end of life" and in cases where "treatment effects are both substantial in restoring health and sustained over a very long period". Drawing lessons from these previous amendments, we critically appraise NICE's proposals. The proposals repeal "end of life" considerations but add consideration of the "proportional" and "absolute" quality-adjusted life-year (QALY) loss from illness. NICE's cost-effectiveness threshold may increase from $£ 20,000$ to $£ 50,000$ per QALY on the basis of these and four other considerations: the "certainty of the ICER [incremental costeffectiveness ratio]"; whether health-related quality of life is "inadequately captured"; the "innovative nature"
\end{abstract}

of the technology; and "non-health objectives of the NHS". We demonstrate that NICE's previous amendments are flawed; they contain logical inconsistencies which can result in different values being placed on health gains for identical patients, and they do not apply value weights to patients bearing the opportunity cost of NICE's recommendations. The proposals retain both flaws and are also poorly justified. Applying value weights to patients bearing the opportunity cost would lower NICE's threshold, in some cases to below $£ 20,000$ per QALY. Furthermore, this baseline threshold is higher than current estimates of the opportunity cost. NICE's proposed threshold range is too high, for empirical and methodological reasons. NICE's proposals will harm the health of unidentifiable patients, whilst privileging the identifiable beneficiaries of new health technologies.
M. Paulden $(\bowtie) \cdot$ C. McCabe

Department of Emergency Medicine, University of Alberta,

736 University Terrace, 8303112 St, Edmonton,

AB T6G 2T4, Canada

e-mail: paulden@ualberta.ca

J. F. O’Mahony

Department of Health Policy and Management,

Trinity College Dublin, Dublin, Ireland

\section{A. J. Culyer}

Centre for Health Economics, University of York,

Heslington, UK

\section{A. J. Culyer}

Institute of Health Policy, Management and Evaluation,

University of Toronto, Toronto ON, Canada 


\section{Key Points for Decision Makers}

The UK's National Institute for Health and Care Excellence (NICE) recently proposed amendments to its methods for the appraisal of new health technologies.

The proposals would increase the upper range of NICE's cost-effectiveness range from $£ 30,000$ to $£ 50,000$ per quality-adjusted life-year (QALY) for all interventions, on the basis of special considerations: "proportional" and "absolute" QALY loss from illness; "certainty of the ICER [incremental cost-effectiveness ratio]"; whether health-related quality of life is "inadequately captured"; the "innovative nature" of the technology; and "non-health objectives of the NHS".

NICE's proposals are problematic: there are inconsistencies in the treatment of social values; the special considerations are unquantified and unjustified; and the proposed threshold range is too high, for both empirical and methodological reasons.

If implemented, the proposals would be destructive to population health, harming unidentified patients in order to privilege the identified beneficiaries of new health technologies.

\section{Introduction}

The UK's National Institute for Health and Care Excellence (NICE) recently proposed amendments to its methods for the appraisal of health technologies [1]. These are based upon the "terms of reference" issued to NICE by the UK's Department of Health, following the UK government's response to the 2013 Health Select Committee report into NICE [2]. The Department of Health called for a number of modifications to NICE's methods to allow for "value assessment of branded medicines under Value-Based Pricing [VBP]", and specifically requested that NICE's methods should, among other requirements:

1. "Include a simple system of weighting for burden of illness that appropriately reflects the differential value of treatments for the most serious conditions";

2. "Include a proportionate system for taking account of wider societal benefits";

3. "Not include a further weighting for therapeutic innovation and improvement"; and

4. "Adopt the same benefit perspective for all technologies falling within the scope of VBP, and for displaced treatments" [3].
In response, NICE issued a consultation paper in March 2014 setting out proposals to amend its existing "Guide to the Methods of Technology Appraisal" [4]. The consultation paper clarifies that NICE currently adopts a baseline cost-effectiveness threshold of $£ 20,000$ per quality-adjusted life-year (QALY), representing the "opportunity cost of programmes displaced by new, more costly technologies" (p. 27). This threshold may be increased up to $£ 30,000$ per QALY upon consideration of four factors: "certainty of the ICER [incremental cost-effectiveness ratio]"; "HRQoL [health-related quality of life] inadequately captured"; "innovative nature of technology"; and "non-health objectives of the NHS" (p. 5). The threshold may be further increased up to $£ 50,000$ per QALY for technologies providing "life extending treatment at the end of life" (p. 5), which were given special consideration by NICE in a 2009 amendment to its guidance [5]. A figure on p. 5 separates this final consideration from the others, implying that the first four together cannot increase the threshold by more than $£ 10,000$ per QALY, while the "end of life" consideration cannot increase the threshold by more than an additional $£ 20,000$ per QALY.

The consultation paper then details NICE's proposed amendments. Consideration of "life extending treatment at the end of life" would be repealed and two new considerations would be added that might justify an increased threshold: "burden of illness" and "wider societal impact". The former is determined by the "proportional QALY loss" resulting from illness, while the latter is proxied by the "absolute QALY loss", which in both cases are calculated from the present time forwards rather than from the onset of illness [6, 7]. Since the proportional QALY loss increases towards 1 as death approaches, "burden of illness" may be viewed as approximating the role of "life extending treatment at the end of life" [7]. Meanwhile, the "wider societal impact" consideration favours the young and/or severely ill, for whom the absolute QALY loss tends to be greatest. The proposed amendments maintain a maximum threshold of $£ 50,000$ per QALY and retain consideration of "certainty of the ICER", "HRQoL inadequately captured", "innovative nature of technology", and "non-health objectives of the NHS". However, the wall of separation between these and other considerations has been removed, along with the $£ 30,000$ per QALY cap on the threshold that may be justified by these four considerations alone (p. 13). Instead, these considerations will be grouped alongside "burden of illness" and "wider societal impact", and collectively these may be used to justify a threshold anywhere between $£ 20,000$ and $£ 50,000$ per QALY (p. 13).

Curiously, the consultation makes no mention of a 2011 NICE guidance amendment, whereby it lowered its discount rate on health effects in cases where "treatment 
effects are both substantial in restoring health and sustained over a very long period", in effect lowering a technology's ICER and increasing the likelihood of adoption [8]. In common with the newly proposed "wider societal impact" consideration, this amendment favoured the young and/or severely ill; indeed, it was implemented specifically so that NICE could recommend an expensive drug for young osteosarcoma patients [9]. Since NICE's consultation does not propose repealing this amendment, NICE's future methods may therefore favour some young and/or severely ill patients in two complementary ways: first by reducing the ICER of treatments through "selective discounting"; and second by allowing for a higher threshold due to "wider societal impact" (and possibly also other considerations).

The purpose of this paper is to appraise NICE's proposals with respect to the consistency of its treatment of social values. First, we review the two previous amendments to NICE's methods, and describe a number of inconsistencies regarding the incorporation of social values in each. We demonstrate these by considering a number of examples in which application of the social values incorporated within NICE's amended guidance results in inconsistent outcomes, including discrimination against the very patients NICE's guidance is intended to benefit. We show that it is not possible for NICE to prioritize some patients without deprioritizing others, and that this deprioritization is not obvious. We also demonstrate that NICE's use of arbitrary criteria in these previous amendments results in discontinuities in NICE's application of social values, with very different values assigned to similar health outcomes for similar patients. Next, we appraise NICE's most recent proposals and consider whether these inconsistencies, or any other issues, are present. We finish by recommending some steps that NICE could take to ensure consistency in its consideration of social values in the future.

\section{Previous Amendments to NICE's Guidance}

Two previous amendments to NICE's guidance focused upon considerations of social value: the 2009 "end of life" amendment, and the 2011 "selective discounting" amendment [5, 8]. Prior to these amendments, NICE's guidance recommended that consistent methods be adopted across all cost-effectiveness analyses [10]. NICE's committees were instructed to use a threshold range of $£ 20,000-£ 30,000$ per QALY in all appraisals, which was intended to represent (in principle) an unmodified estimate of the opportunity cost of adopting technologies within a fixed NHS budget [10, 11]. Future costs and health outcomes were discounted at a single rate in all appraisals. Overall, NICE's methods broadly reflected a basic equity position in which each QALY was assigned equal value for all individuals in society (the so-called "a QALY is a QALY" position). Despite concerns raised by Harris and others [12], NICE's methods did not inherently discriminate on the basis of life expectancy (LE) [13].

\section{1 "End of Life" Amendment (2009)}

NICE's "end of life" amendment marked a change in this basic equity position. It specified the following criteria which justified giving "greater weight to QALYs achieved in the later stages of terminal diseases" when appraising "end of life treatments":

- "The treatment is indicated for patients with a short life expectancy, normally less than 24 months";

- "There is sufficient evidence to indicate that the treatment offers an extension to life, normally of at least an additional 3 months, compared to current NHS treatment"; and

- "The treatment is licensed or otherwise indicated, for small patient populations" [5].

Where these criteria apply, NICE's appraisal committees were to consider "the magnitude of the additional weight that would need to be assigned to the QALY benefits in this patient group for the cost-effectiveness of the technology to fall within the current threshold range" [5]. However, NICE's recent consultation notes that, rather than assigning an additional weight to QALY benefits, NICE reinterpreted this amendment as permitting a higher threshold of up to $£ 50,000$ per QALY, regarded as equivalent to applying "a maximum weight of 2.5 from a starting point of $£ 20,000$ per QALY" [4]. Since, at the time of this amendment, NICE's best estimate of the opportunity cost of its decisions was reflected by its threshold range of $£ 20,000-£ 30,000$ per QALY, its willingness to recommend "end of life" treatments with ICERs of up to $£ 50,000$ per QALY implied that NICE no longer valued the QALYs of all individuals equally; instead, providing an additional QALY to an "end of life" patient was assigned approximately twice the value of providing an additional QALY to any other patient. As Paulden and Culyer [13] noted, this increased the potential for NICE's guidance to discriminate against patients with longer LE.

\subsubsection{Inconsistencies Resulting from the Use of Arbitrary Cut-Offs}

Although NICE's methods are constructed around the use of the QALY as a measure of effectiveness, the cut-offs specified in the "end of life" amendment are based upon 
unadjusted LE: typically patients must have "less than 24 months" of remaining LE and be the beneficiary of a treatment appraised by NICE that "offers an extension to life, normally of at least an additional 3 months, compared to current NHS treatment" [5]. Thus a treatment for a patient with 3 years of remaining LE of poor quality would not meet the "end of life" criteria, while a treatment for another patient with 18 months of remaining LE of excellent quality might meet them, even if the first patient has fewer remaining QALYs. Similarly, a treatment providing an additional 3 months LE of extremely poor quality might satisfy the criteria, while a treatment providing an additional 2 months LE of excellent quality would not, even if the latter provided a greater QALY benefit. It is not clear why NICE regards quality of life as integral to decisions regarding cost-effectiveness, yet irrelevant to its "end of life" criteria.

In cases where a technology satisfies NICE's "end of life" criteria by meeting the 3-month cut-off, NICE applies an additional weight to all of the health benefits gained, not only to those health benefits experienced beyond the cutoff. The perversity of this is best shown by example. Suppose NICE appraises a technology (A) which provides an additional 2 months of LE of a given quality and which otherwise meets the "end of life" criteria. Since the technology fails to meet the 3-month cut-off, no additional weight is applied to patients' QALYs. Now suppose NICE appraises a similar technology (B) for the same patient subgroup which provides 3 months of additional LE of a slightly lower quality than that of technology A. Since technology B meets the "end of life" criteria, NICE would apply a weight to all of the QALYs gained by its beneficiaries, including those gained during the first 2 months of extended LE. For those 2 months, NICE may therefore apply a higher value to a lower quality state of health for exactly the same patients - the very patients NICE's "end of life" amendment intended to benefit.

\subsubsection{Inconsistencies Resulting from the Failure to Consider Opportunity Cost}

Because disinvestment decisions in the NHS are taken by local decision makers, NICE does not know which specific services will be displaced following its recommendations. Nevertheless, given that a substantial proportion of health care resources are used by patients who are approaching death, at least some of this opportunity cost must fall upon patients regarded as being at the "end of life" [14]. When NICE recommends a new "end of life" treatment, many of the patients bearing the opportunity cost will therefore be similar to those who stand to benefit. If NICE places a greater value on the health of "end of life" patients, it follows that they must account for those similarly placed patients bearing the opportunity cost. However, the "end of life" amendment only places a greater value on the health of the beneficiaries of treatment under review.

While it may seem appropriate to use a higher threshold to account for a greater value placed on the health of the beneficiaries of treatment (an assumption returned to below), there are important implications for the threshold when we consider how a greater value on health might apply to those bearing the opportunity cost. When a greater value is applied to displaced health, this implies that a lower threshold should be used. The appropriate threshold depends upon the proportion of those bearing the opportunity cost considered to be at the "end of life" and therefore deserving of special consideration. Given the increasing data on the characteristics of the recipients of NHS care, the resulting threshold can and should be evidence based [15].

Suppose that NICE is appraising a new treatment for end of life patients, and assume (for now) that the opportunity cost of adopting the treatment is known to fall entirely upon existing services for patients also at the end of life. Suppose that for every $£ 20,000$ spent on the new treatment, a single QALY is forgone by displacing existing services, i.e. the shadow price of the relevant budget is $£ 20,000$ per QALY. Finally, suppose that NICE wishes to assign $2.5 \times$ the value to additional QALYs for end of life patients as it does to additional QALYs for all other patients. What threshold should NICE use to appraise the new end of life treatment? Following the logic of its "end of life" amendment and subsequent implementation, NICE would adopt a $£ 50,000$ per QALY threshold. Yet this would be counterproductive, because a new treatment with an ICER of $£ 40,000$ per QALY would displace two QALYs for each QALY gained, and those displaced QALYs would be forgone by end of life patients whose health should also be valued $2.5 \times$ as highly. Under such guidance, NICE would recommend some new treatments with ICERs above $£ 20,000$, even though these would displace more QALYs than they gain in end of life patients, the very group NICE ostensibly values more. It logically follows that where every patient is subject to special consideration-including the beneficiaries of treatment and those bearing the opportunity cost - the appropriate threshold to adopt is $£ 20,000$ per QALY, exactly the same as the shadow price of the budget. Alternatively, suppose that none of the opportunity cost falls on end of life patients, but rather on other patients not considered to be at the end of life. In this case, the appropriate threshold to adopt is indeed $£ 50,000$ per QALY. Evidently, a far more realistic assumption would be that some of those patients bearing the opportunity cost are subject to special consideration but others are not. In this case, the appropriate threshold lies somewhere between $£ 20,000$ and $£ 50,000$ per QALY. The greater the proportion of end of life patients among those bearing the opportunity cost, the closer the threshold should be to $£ 20,000$ per QALY. 
NICE's decision to assign special consideration to "end of life" patients also has important implications beyond the appraisal of "end of life" treatments. Even when NICE appraises a new technology that does not meet the "end of life" criteria, the potential exists for its opportunity cost to be borne by patients who are at the end of life. Returning to the example above, suppose (for now) that none of the beneficiaries of the technology, but all of the patients bearing the opportunity cost, are considered to be at the end of life and subject to special consideration. One QALY is forgone by end of life patients for every $£ 20,000$ spent on the new technology, and each forgone QALY is assigned twice the value of each QALY gained. It follows that the appropriate threshold is $£ 10,000$ per QALY. Alternatively, and more realistically, if only some of those bearing the opportunity cost are subject to special consideration, then the appropriate threshold lies somewhere between $£ 10,000$ and $£ 20,000$ per QALY, depending upon the proportion of those bearing the opportunity cost who are subject to special consideration. The critical point is that, by assigning special consideration to one subgroup of patients (in this case, those at the "end of life"), NICE must use a threshold lower than the shadow price of the budget when appraising technologies that do not benefit this subgroup.

Since NICE's subsequent amendments have broadened the scope for patients to be assigned special consideration beyond "end of life" cases, it is useful to specify generalizable results:

i. The greater the weight placed on the health of those provided special consideration, and the greater the proportion of such patients among those bearing the opportunity cost of NICE's recommendations, the lower the threshold NICE should use in its appraisal of technologies which do not benefit such patients.

ii. Where multiple avenues exist for assigning special consideration (as under NICE's recently proposed amendments), if the bearers of the opportunity cost are assigned greater special consideration than the beneficiaries of treatment then the threshold should be lower than the shadow price of the budget, and vice versa.

Three critical results follow from this:

1. The greater the scope for NICE to assign special consideration to patients, the lower the threshold must be for technologies that benefit patients not assigned special consideration, since patients given special consideration will constitute a greater proportion of those bearing the opportunity cost.

2. If the case mix of those benefitting from technologies recommended by NICE is similar to the case mix of those bearing the opportunity cost, then the weighted average of the thresholds used across all of NICE's appraisals must equal the shadow price of the budget, where this average is weighted by the budget impact of each technology appraised.

3. If NICE specifies a maximum weight that may be assigned to the health of any patient (as it does in its recent proposals), and if any of those bearing the opportunity cost are assigned special consideration, then the maximum threshold that NICE may use for any appraisal is unambiguously lower than the product of this weight and the shadow price of the budget.

It follows that NICE's current and proposed threshold range is too high; the maximum threshold of $£ 50,000$ per QALY is too high in all cases — even when appraising "end of life" treatments—and the minimum threshold of $£ 20,000$ per QALY is also too high in many cases. As a result, NICE may be recommending new treatments which displace not only more QALYs but also more value than they provide, privileging the identifiable beneficiaries of new interventions recommended by NICE while harming the unidentified users of existing NHS services who bear the opportunity cost of their adoption.

\subsubsection{Inconsistencies Resulting from the Conflation of QALY Weights and Threshold Weights}

Although NICE's "end of life" amendment requires appraisal committees to consider "the magnitude of the additional weight that would need to be assigned to the QALY benefits" in order for an "end of life" treatment to appear cost-effective, NICE has reinterpreted this as permitting a higher threshold of $£ 50,000$ per QALY, corresponding to a weight of 2.5 applied to a $£ 20,000$ per QALY threshold. However, as demonstrated above, if any of the patients bearing the opportunity cost are also granted special consideration, then the appropriate threshold is not a simple multiple of the shadow price of the budget and the QALY weight.

Even if none of those patients bearing the opportunity cost is given special consideration, the reinterpretation of QALY weights as a threshold weight is problematic. Consider a treatment which costs less than its comparator and is less effective (i.e. it lies in the south west quadrant of the cost-effectiveness plane). The treatment should be considered cost-effective only if its ICER lies above the threshold, and a weight on the QALYs of the beneficiaries should be accounted for by lowering the threshold rather than raising it.

Next, consider a treatment that is more expensive but less effective, or vice versa (i.e. it lies in the north west or south east quadrant). If a higher weight is applied to the QALYs of the beneficiaries, this will move the treatment deeper into its respective quadrant (Fig. 1). This is clearly of interest to NICE, since this will reduce uncertainty about whether the treatment is cost-effective. Yet there is no means to account for this by adjusting the threshold. 
Finally, consider a new treatment for "end of life" patients with two comparators: usual care, which is less expensive and less effective; and an alternative treatment, which is less expensive but more effective. Suppose the alternative treatment provides an additional 2 months of LE compared with usual care at a greatly improved HRQoL, whereas the new treatment provides 4 months of additional LE compared with usual care but at a worsened HRQoL. Only the new treatment meets NICE's "end of life" criteria. Suppose that, when a weight is placed on the QALYs of the beneficiaries of the new treatment, it now appears both more effective and more cost-effective than the alternative treatment (Fig. 2). If NICE were to apply this weight to the threshold instead of the QALYs directly, then the new treatment would appear to be dominated by the alternative treatment and hence appear (incorrectly) to be not cost-effective.

It follows that the use of a threshold weight rather than a QALY weight may result in inconsistencies when

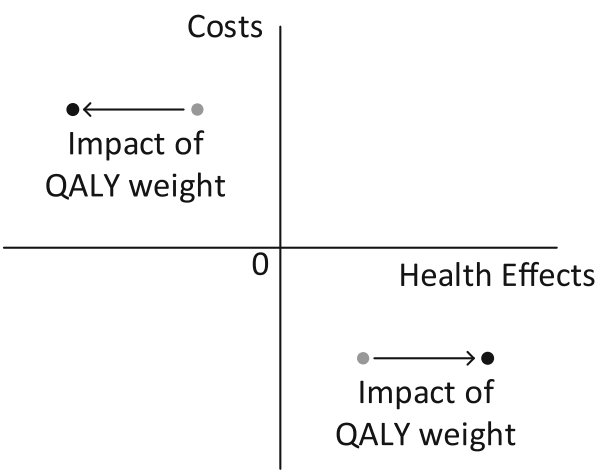

Fig. 1 Potential impact of applying QALY weights to strategies in the north west and south east quadrants. $Q A L Y$ quality-adjusted lifeyear

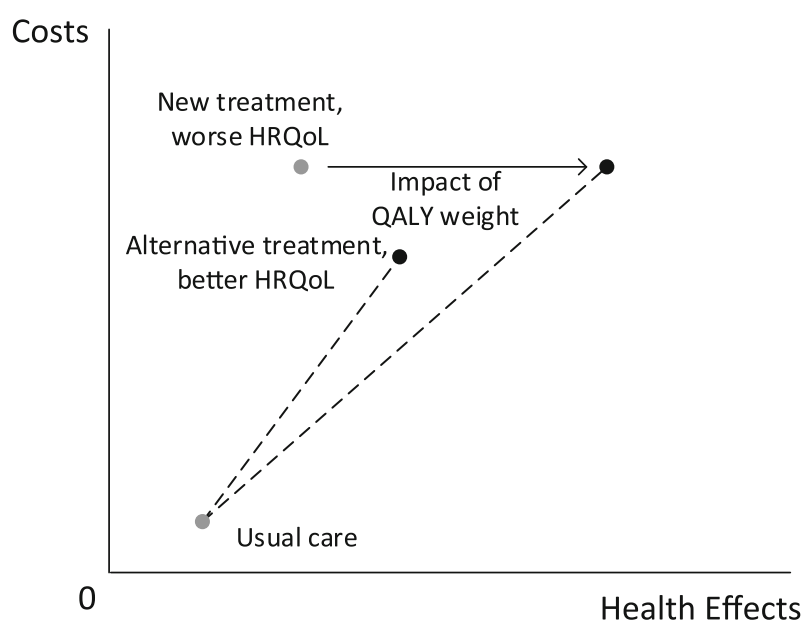

Fig. 2 Potential impact of applying QALY weights to a dominated strategy in the north east quadrant. $H R Q o L$ health-related quality of life, $Q A L Y$ quality-adjusted life-year appraising technologies with more than one comparator and/or which lie outside of the north east quadrant of the cost-effectiveness plane. A solution to these difficulties is to adopt a "net benefit" framework in which both health benefits and the health expected to be forgone can be weighted directly for each strategy [16].

\section{2 "Selective Discounting" Amendment (2011)}

In 2011, NICE made a further amendment to its methods guidance alongside its appraisal of mifamurtide, a drug indicated for osteosarcoma (a rare disease that principally afflicts children and young adults) [8]. Under NICE's standard $3.5 \%$ per annum discount rate, mifamurtide's estimated ICER was $£ 57,000$ per QALY. The appraisal committee noted that applying differential discounting, at 3.5 and $1.5 \%$ for costs and health effects, respectively, reduced the ICER to $£ 36,000$ per QALY. NICE amended its guidance to state that costs and health effects be differentially discounted at 3.5 and $1.5 \%$, respectively, in selective cases in which "treatment effects are both substantial in restoring health and sustained over a very long period (normally at least 30 years)". Following this amendment, NICE recommended mifamurtide.

O'Mahony and Paulden [9] outlined a number of concerns and inconsistencies with this amendment. Among these was the increased scope for NICE's guidance to discriminate on the basis of LE, since the arbitrary "30 years" cut-off excludes individuals with less than 30 years LE following treatment. In NICE's 2013 “Guide to the Methods of Technology Appraisal", the lower $1.5 \%$ rate was also applied to costs [17]. While this satisfied one of the concerns expressed by O'Mahony and Paulden, other inconsistencies remained unaddressed.

\subsubsection{Inconsistencies Resulting from the Use of Arbitrary Cut-Offs}

In common with the "end of life" amendment, the criteria for NICE's "selective discounting" amendment use an arbitrary cut-off: a technology should provide a treatment effect for "at least 30 years". If a technology meets these criteria, a lower discount rate is applied to health benefits in all time periods, not only those after the cut-point. As O'Mahony and Paulden note, this results in potential inconsistencies. Consider two interventions for the same patients, the first yielding benefits for 29 years, the second yielding slightly smaller benefits for 29 years and an additional benefit in the 30th year (and so only the second meets the criteria for selective discounting). Since NICE would apply a lower discount rate to benefits from the second intervention in all 30 years, for the initial 29 years, NICE may assign a 
higher value to a lower quality state of health for exactly the same patients. As in the example from NICE's "end of life" amendment provided earlier, this would harm the very patients the amendment was intended to benefit.

\subsubsection{Inconsistencies Resulting from the Failure to Consider Opportunity Cost}

As with the "end of life" amendment, the "selective discounting" amendment fails to consider that similar (or identical) patients to those granted special consideration among the beneficiaries of treatment might bear the opportunity cost of NICE's recommendations. While a solution to this inconsistency in the case of the "end of life" amendment is to reduce the threshold, accounting for opportunity cost within a "selective discounting" framework is not straightforward.

Suppose that the appropriate discount rate for costs and health benefits in "non-special" cases is $3.5 \%$, and that NICE wishes to give special consideration to some patients by applying a lower $1.5 \%$ discount rate to their health outcomes. It follows that a lower discount rate should also be applied to the health outcomes forgone by those patients subject to special consideration who bear the opportunity cost. Although these benefits forgone are not accounted for directly in the ICER, discounting the health benefits forgone at a lower rate is equivalent to discounting the incremental costs of the technology at a lower rate (assuming the shadow price of the budget remains constant) $[16,18]$. In cases where every patient benefiting from treatment and every patient bearing the opportunity cost is subject to special consideration, the same lower discount rate may simply be applied to both the incremental costs and incremental health benefits. But if only a proportion of patients who bear the opportunity cost are subject to special consideration, then incremental costs should be discounted at a rate somewhere between 1.5 and $3.5 \%$ (depending upon this proportion). Furthermore, in cases where the beneficiaries of treatment are not subject to special consideration, the potential still exists for some patients bearing the opportunity cost to be subject to special consideration. In such cases, incremental benefits should be discounted at $3.5 \%$ and incremental costs at a rate between 1.5 and $3.5 \%$. It follows that neither the original nor the modified amendment appropriately accounted for opportunity cost. It also seems far more straightforward and transparent for NICE to assign a direct weight to the QALYs of patients provided with special consideration than to use "selective discounting".

\section{The Proposed Amendment to NICE's Guidance}

The proposals in NICE's consultation suffer from many of the same inconsistencies afflicting NICE's previous amendments. There are specific flaws with the conditions attached to QALY weightings that are analogous to specific flaws with previous amendments. There is also a general flaw in all of NICE's amendments that special considerations are not applied consistently across the beneficiaries and those bearing the opportunity cost.

\subsection{Issues Arising from the Use of "Absolute QALY Loss" as a Proxy for "Wider Societal Impact"}

NICE was asked by the Department of Health to consider the "wider social impact" associated with a disease; however, it is unclear how this is related to the proposed weighting of "absolute QALY loss", i.e. the health lost by individuals. Considering wider societal impact risks prioritization of those with greater economic or social participation, since restoring the health of such individuals may be associated with greater productivity gains than restoring the health of other individuals. This would appear to be in contravention of the NHS Constitution, which states that "access to NHS services is based on clinical need, not an individual's ability to pay" $[19,20]$. This can be mitigated by applying a common productivity weight to all individuals; however, if the number of beneficiaries and patients bearing the opportunity cost is equal, then decisions will be unaffected. It follows that accounting for wider social impact is either unlawfully discriminatory or potentially unnecessary.

\subsection{Inconsistencies in Weighting Disease Severity from the Use of "Absolute QALY Loss"}

The proposed weight for "absolute QALY loss" assigns greater value to treatments for diseases that impose larger QALY losses over a patient's lifetime, irrespective of the health gain per unit of expenditure. This can result in inconsistencies whereby individuals with a disease that persists continuously over many years will benefit from a higher weighting on their health than otherwise similar individuals with multiple independent diseases that impose the same total QALY loss. This may serve to bias health care resource allocation in favour of chronic disease management in a way that would not be justified by an objective of maximizing health gain. Furthermore, it potentially introduces discrimination between patients that have similar capacity to benefit from health care expenditure. It may also result in age-based discrimination; since the absolute QALY loss from a disease tends to be greater with longer remaining LE, and since younger patients usually have longer LE, the absolute QALY weighting stands to favour the young over the old irrespective of their potential health gain per unit of expenditure. 


\subsection{Inconsistent Treatment of Benefits Due} to Consideration of "Proportional QALY Loss"

The proposed weighting for "proportional QALY loss" also creates potential for inconsistencies in the weighting of health effects. The proportional QALY loss depends upon the remaining LE without the disease in question, generally resulting in a smaller weighting for younger patients. A common health gain - for example, the treatment of an acute event without long-term health effects-may therefore be weighted differently for young and old patients. It is doubtful if the potential biases of the proportional and absolute QALY loss weights will systematically compensate in a way to allay concerns of age discrimination becoming inherent in NICE's decision making process.

\subsection{Inconsistencies Resulting from Capping the Threshold Weight at $2.5 \times$}

NICE's proposed limit of $2.5 \times$ on the weight that can be applied to the baseline $£ 20,000$ per QALY threshold introduces an apparent inconsistency whereby special considerations may carry more value when applied to independent interventions than when applied simultaneously to a common intervention. Consequently, NICE is advocating explicitly allocating additional resources in response to the presence of specific attributes in some circumstances, but not rewarding the very same attributes in other circumstances. This inconsistency stands to create inefficiencies and potentially unwarranted discrimination between otherwise similar patients.

\subsection{Inconsistencies Between NICE's Threshold and Empirical Estimates of the Opportunity Cost}

Despite acknowledging that the baseline cost-effectiveness threshold of $£ 20,000$ per QALY represents the "opportunity cost of programmes displaced by new, more costly technologies", NICE makes no mention of the extensive recent empirical work — supported by NICE_-which aimed to estimate this [21]. This work estimated the shadow price of the NHS budget to be below $£ 20,000$ per QALY, implying that NICE's proposed threshold is too high and is likely to result in the adoption of technologies which displace more value than they create.

\subsection{Inconsistencies Resulting from the Failure to Consider Opportunity Cost}

As with previous amendments, NICE's proposals do not apply value considerations consistently to the beneficiaries of new technologies and those who bear the opportunity cost. NICE is proposing to adopt a higher threshold for appraising new technologies depending upon the "certainty of the ICER", whether HRQoL is "inadequately captured", the "innovative nature" of the technology, and "non-health objectives of the NHS", yet the impact upon each of these from the displacement of existing services will not be considered. Indeed, if a special weight were to be attached to "certainty of the ICER" for both the new technology and the opportunity cost, this might be expected to raise the value of existing services relative to new technologies, because of the greater certainty of the costs and effectiveness of displaced services arising from their use in practice.

\section{Discussion}

The recently proposed amendments to NICE's guidance raise a considerable number of concerns. NICE is proposing a formal system for assigning values to health benefits using weights that are neither explicitly stated nor consensus based. The quantitative basis for these weights has neither been provided nor evidenced; while NICE has applied implicit weights to certain attributes in the past, this is not a sound rationale for applying such weights in the future. Although the proposed system of weights ostensibly offers a formalization of NICE's decision criteria, the criteria remain in large part arbitrary and opaque. In essence, the proposals extend the limit of the threshold range for non-"end of life" treatments from $£ 30,000$ to $£ 50,000$ per QALY, increasing the scope for unaccountable discretion and allocations that are neither efficient nor fair.

The proposals also raise a number of questions. Is NICE's favouring of the young, those with severe illness, and individuals at the end of life consistent with the values of the UK public? Why has NICE proposed to repeal the "end of life" amendment but not the "selective discounting" amendment, given that the effect of each is approximated through the new "burden of illness" and "wider societal impact" considerations? Why do the proposals extend the scope for the threshold to be increased due to "innovative nature of technology" when the Department of Health's terms of reference specifically request that NICE "not include a further weighting for therapeutic innovation and improvement"? And why has NICE failed to apply special value weights to those bearing the opportunity cost of its decisions, despite the Department of Health's request that NICE "adopt the same benefit perspective for all technologies... and for displaced treatments"?

Within a resource-constrained health care system, it is not possible to improve treatment access for one group of individuals without curtailing access for other groups. NICE's apparent favouring of the young and those at the 
end of life inevitably disadvantages other patients. Even if discrimination on such grounds is consistent with the values of the public, NICE's proposed methods are not. NICE has repeatedly privileged the identified beneficiaries of treatment over those bearing the opportunity cost. As a result, NICE may recommend a treatment which displaces more QALYs than it gains in the very patients whose health it ostensibly values more. This may create the perception that NICE does not value the special value considerations per se, but only if doing so favours the adoption of a new technology. Such an approach would be ethically untenable as well as manifestly incompatible with NICE's previous basic equity position and the terms of reference provided by the Department of Health.

This raises the broader issue of whether NICE's revealed values are defensible-specifically, valuing the health of some patients more than others. It might be argued that NICE is an agent of a legitimate and accountable higher authority (the UK's elected parliament), and so its values should be those that prevail [16, 22]. Or it might be held that it is the values of the British public, perhaps as revealed by NICE's Citizens Council, that should be reflected in NICE's methods. It is not clear to which possible source of moral authority these various amendments are appealing, nor which would be the more legitimate. Further, it is not apparent whether NICE's interpretation of these unclearly expressed values is reasonable. What is evident, however, is that an inconsistent treatment of social values cannot be sustained. It may therefore be timely for NICE to hold back from a poorly evidenced incorporation of social value arguments in its decision making processes while better evidence is generated regarding the values held by the public and also by social agents. It may be informative to test, for example, the extent to which NICE's previous basic equity position ("a QALY is a QALY") is generally acceptable, and what exceptions (if any) might be widely accepted by the public. The value judgments of policy makers well-versed in seeking solutions that transcend sectional interests may also be revealed through well-conducted experiments (the subjects of which may include parliamentarians and the members of NICE's Appraisal Committees). An appropriate strategy for NICE at this stage might therefore be to seek National Institute for Health Research support for such work.

In light of this, we recommend that NICE should:

1. Eliminate arbitrary cut-offs in the application of value weights;

2. Implement research and public consultation processes to support the development of a broader value framework and associated implementation plans. This would require that NICE: (a) Specify how it will operationalize the measurement of each of the special value considerations included in the revised methods;

(b) Specify the magnitude of the value weight it will assign to each special value consideration, and the evidence on which that weight is based;

(c) Specify how the value weights assigned to all the special value considerations will be aggregated to arrive at the 'value multiplier' for each specific technology appraisal;

(d) Specify how it will operationalize the assessment of the special value considerations in the patient groups likely to bear the opportunity cost of its recommendations, in order to meet the requirement that it "adopt the same benefit perspective for all technologies... and for displaced treatments".

Satisfying these recommendations will not be straightforward. An expert workshop may be worth convening to resolve the issues we have raised, and, so far as possible, to achieve consensus on future revisions. NICE's accretion of ad hoc adjustments has compounded inconsistency upon inconsistency and, quite apart from being inherently undesirable, the lack of transparency has made it hard for ordinary people to understand NICE's reasoning. It is plain that the current proposals are unlikely to command agreement, not because of disagreement with NICE's social value judgments, but because of the inappropriate way in which it treats people having the same characteristics, and hence entitlements, differentially. There is a fairly straightforward remedy for all of these difficulties, whose starting point is to address priorities by attributing weights to those whom NICE wishes especially to protect, rather than by adjusting discount rates or thresholds. Further research relating to those bearing the opportunity cost, and the prevalence of special characteristics amongst them, is required to give reasonable effect to this symmetry of treatment.

Until such research is complete, we recommend that NICE reverts to the basic equity position it adopted prior to the recent amendments, under which all QALYs were assigned equal value ("a QALY is a QALY"). Not only would this reduce the scope for discrimination on the basis of LE, but it would give all patients greater confidence that NICE has consistently considered the impact of its recommendations on their health. It would also satisfy the Department of Health's requirements that NICE "adopt the same benefit perspective for all technologies... and for displaced treatments" and also "not include a further weighting for therapeutic innovation and improvement", neither of which is satisfied by NICE's recent proposals.

It might be argued that reverting to equal value weights would preclude the use of "a simple system of weighting 
for burden of illness that appropriately reflects the differential value of treatments for the most serious conditions" or "a proportionate system for taking account of wider societal benefits", both of which were also requested by the Department of Health. However, if NICE calculates QALYs using an EQ-5D utility algorithm with an N3 term, which provides a weight for the added disutility of severe ill health on one or more dimensions, then this alone might meet the requirement to account for the burden associated with severe illness through a "simple system of weighting" [23]. This approach has the added attraction of being derived from a large survey of UK public values. Furthermore, as we noted earlier, it does not appear possible to account for "wider societal benefits" in a way that would make a difference to NICE's decision making while also remaining consistent with the principles laid out in the NHS Constitution. Since an unlawful system for taking account of wider societal benefits is clearly not "proportional", it may therefore not be feasible for NICE to meet this specific request. It follows that reverting to its previous basic equity position, under which all QALYs were assigned equal value, may be the most appropriate means for NICE to satisfy, in the short term and to the greatest extent possible, the requirements placed upon it by the Department of Health.

Our critique of NICE's proposals should be tempered by an acknowledgment that NICE was placed in a difficult position; it was obliged to modify its methods in a way that was unlikely to be achieved by consensus. Nevertheless, it should be noted that NICE's proposals allow for large additional QALY weights. As such, NICE's proposals do not seem to be a conservative response to the requests made of it. Furthermore, NICE's proposals do not meet the Department of Health's requirements: they fail to apply the same benefits perspective adequately to health displaced, they maintain a further weighting for therapeutic innovation and improvement, and the absolute QALY loss adjustment does not clearly correspond to "wider social impact".

It was a notable feature of the early years of NICE that difficult questions of method were identified openly so that all who might claim to have relevant expertise were able to participate fully in both the creation of, and subsequent revisions to, the methods guidance. We do not doubt that such transparency continues to be a prime social value of NICE.

Acknowledgments The authors wish to thank Richard Edlin for providing insightful comments during discussions prior to the preparation of this manuscript. All authors contributed towards the ideas presented in this paper, and all authors assisted with the writing and editing of the manuscript. AJC was Vice Chair of NICE from 1999 to 2003 and is currently Chair of NICE's International Advisory Group, but does not consider himself to be conflicted for the purposes of this manuscript. CM was Director of NICE's Decision Support Unit from
2003 to 2006, and a member of NICE's Medical Technologies Advisory Committee from 2010 to 2011, but does not consider himself to be conflicted for the purposes of this manuscript. MP is supported by grants from the Canadian Institutes of Health Research (CIHR) and Genome Canada. JOM is supported by Ireland's Health Research Board. CM is supported by a Capital Health Research Chair Endowment. MP and JOM have no conflicts of interest. MP is the overall guarantor of this work.

\section{References}

1. National Institute for Health and Care Excellence (NICE). Methods of technology appraisal consultation. 2014. Available from: https://www.nice.org.uk/Guidance/InConsultation/GIDINCONSULTATION/html/p/methods-of-technology-appraisalconsultation?id=2cbiqn4bjozoxf4h6trcemndea.

2. Secretary of State for Health. The Government's Response to the Health Select Committee's Eighth Report of Session 2012-2013 on the National Institute for Health and Clinical Excellence. London; 2013. Available from: https://www.gov.uk/government/ uploads/system/uploads/attachment_data/file/164232/The_Government_s_Response.pdf.

3. Department of Health. Value-based pricing - terms of reference. London; 2013. Available from: http://www.nice.org.uk/proxy/ ?sourceUrl=http $\% 3 \mathrm{~A} \% 2 \mathrm{~F} \% 2 \mathrm{Fwww}$.nice.org.uk\%2Faboutnice\% 2Fhowwework\%2Fdevnicetech\%2FDevelopmentWorkForValue BasedPricing.jsp\%3Fdomedia\%3D1\%26mid\%3D9A46903F-A8 A3-2090-57684CA0D69C6E1D.

4. National Institute for Health and Care Excellence (NICE). Consultation paper-value based assessment of health technologies. London; 2014. Available from: https://www.nice.org.uk/ Guidance/InConsultation/GID-INCONSULTATION/html/p/methods-of-technology-appraisal-consultation?id=2cbiqn4bjozoxf4h6tr cemndea.

5. National Institute for Health and Care Excellence (NICE). Appraising life-extending, end of life treatments. London; 2009. Available from: http://www.nice.org.uk/guidance/gid-tag387/ resources/appraising-life-extending-end-of-life-treatments-paper2.

6. Stolk EA, Van Donselaar G, Brouwer WBF, Busschbach JJV. Reconciliation of economic concerns and health policy: illustration of an equity adjustment procedure using proportional shortfall. Pharmacoeconomics. 2004;22:1097-107.

7. Office for Health Economics (OHE). Office for Health Economics note on proportional versus absolute shortfall. London; 2014. Available from: http://www.gserve.nice.org.uk/media/FE2/ C7/OHE_Note_on_proportional_versus_absolute_shortfall.pdf.

8. National Institute for Health and Clinical Excellence (NICE). Discounting of health benefits in special circumstances. London; 2011. Available from: http://www.nice.org.uk/guidance/ta235/ resources/osteosarcoma-mifamurtide-discounting-of-health-benefits -in-special-circumstances2.

9. O'Mahony JF, Paulden M. NICE's selective application of differential discounting: ambiguous, inconsistent, and unjustified. Value Health. 2013;2-4. Available from: http://www. valueinhealthjournal.com/article/S1098-3015\%2813\%2900069-7/ abstract.

10. National Institute for Health and Clinical Excellence. Guide to the methods of technology appraisal. London: NICE; 2008.

11. McCabe C, Claxton K, Culyer AJ. The NICE cost-effectiveness threshold: what it is and what that means. Pharmacoeconomics. 2008;26:733-44. Available from: http://www.ncbi.nlm.nih.gov/ pubmed/18767894.

12. Harris J. Nice and not so nice. J Med Ethics. 2005;31:685-8. Available from: http://jme.bmj.com/content/31/12/685.full. 
13. Paulden M, Culyer AJ. Does cost-effectiveness analysis discriminate against patients with short life expectancy? Matters of logic and matters of context. Toronto: THETA Collaborative, University of Toronto; 2010. Working Paper 1. Available from: http://theta.utoronto.ca/Working_Papers.

14. Seshamani M, Gray AM. A longitudinal study of the effects of age and time to death on hospital costs. J Health Econ. 2004;23:217-35.

15. Kasteridis P, Street A, Dolman M, Gallier L, Hudson K, Martin J, et al. The importance of multimorbidity in explaining utilisation and costs across health and social care settings: evidence from south Somerset's symphony project. Heslington: Centre for Health Economics, University of York; 2014. CHE Research Paper 96. Available from: http://www.york.ac.uk/media/che/ documents/papers/researchpapers/CHERP96_multimorbidity_ utilisation_costs_health_social\%20care.pdf.

16. Claxton K, Paulden M, Gravelle H, Brouwer W, Culyer AJ. Discounting and decision making in the economic evaluation of health-care technologies. Health Econ. 2011;20:2-15. Available from: http://onlinelibrary.wiley.com/doi/10.1002/hec.1612/full.

17. National Institute for Health and Care Excellence. Guide to the methods of technology appraisal 2013. London; 2013. Available from: http://www.nice.org.uk/article/PMG9/chapter/Foreword.

18. Claxton K, Sculpher M, Culyer A, McCabe C, Briggs A, Akehurst $\mathrm{R}$, et al. Discounting and cost-effectiveness in NICE- stepping back to sort out a confusion. Health Econ. 2006;15:1-4. Available from: http://onlinelibrary.wiley.com/doi/10.1002/hec. 1081/abstract.

19. Raftery J. Value based pricing: can it work? BMJ. 2013;5941: f5941.

20. Department of Health. The NHS constitution. London; 2013. Available from: http://www.nhs.uk/choiceintheNHS/Rightsand pledges/NHSConstitution/Documents/2013/the-nhs-constitutionfor-england-2013.pdf.

21. Claxton K, Martin S, Soares M, Rice N, Spackman E, Hinde S. Methods for the estimation of the NICE cost effectiveness threshold. Heslington: Centre for Health Economics, University of York; 2013. CHE Research Paper 81. Available from: http:// www.york.ac.uk/media/che/documents/papers/researchpapers/CH ERP81_methods_estimation_NICE_costeffectiveness_threshold_ \%28Nov2013\%29.pdf.

22. Paulden M, Claxton K. Budget allocation and the revealed social rate of time preference for health. Health Econ. 2009;21: 612-618. Available from: http://onlinelibrary.wiley.com/doi/10. 1002/hec.1730/full.

23. Dolan P. Modeling valuations for EuroQol health states. Med Care. 1997;35:1095-108. 